\title{
PKC $\lambda$ in liver mediates insulin-induced SREBP-1c expression and determines both hepatic lipid content and overall insulin sensitivity
}

\author{
Michihiro Matsumoto, ${ }^{1}$ Wataru Ogawa, ${ }^{1}$ Kazunori Akimoto, ${ }^{2}$ Hiroshi Inoue, ${ }^{1}$ \\ Kazuaki Miyake, ${ }^{1}$ Kensuke Furukawa, ${ }^{1}$ Yoshitake Hayashi, ${ }^{3}$ Haruhisa Iguchi, ${ }^{4}$ \\ Yasushi Matsuki, ${ }^{4}$ Ryuji Hiramatsu, ${ }^{4}$ Hitoshi Shimano, ${ }^{5}$ Nobuhiro Yamada, ${ }^{5}$ \\ Shigeo Ohno, ${ }^{2}$ Masato Kasuga, ${ }^{1}$ and Tetsuo $\mathrm{Noda}^{6}$
}

\author{
${ }^{1}$ Department of Clinical Molecular Medicine, Division of Diabetes and Digestive and Kidney Diseases, Kobe University \\ Graduate School of Medicine, Kobe, Japan \\ ${ }^{2}$ Department of Molecular Biology, Yokohama City University Graduate School of Medical Science, Yokohama, Japan \\ ${ }^{3}$ Division of Molecular Medicine and Medical Genetics, International Center for Medical Research, Kobe University \\ Graduate School of Medicine, Kobe, Japan \\ ${ }^{4}$ Genomics Science Laboratories, Sumitomo Pharmaceuticals Co. Ltd., Takarazuka, Japan \\ ${ }^{5}$ Department of Internal Medicine, Division of Endocrinology/Metabolism, Institute of Clinical Medicine, \\ University of Tsukuba, Tsukuba, Japan \\ ${ }^{6}$ Department of Experimental Pathology, Cancer Institute, Tokyo, Japan
}

\begin{abstract}
$\mathrm{PKC} \lambda$ is implicated as a downstream effector of PI3K in insulin action. We show here that mice that lack PKC $\lambda$ specifically in the liver (L- $\lambda$ KO mice), produced with the use of the Cre-loxP system, exhibit increased insulin sensitivity as well as a decreased triglyceride content and reduced expression of the sterol regulatory element-binding protein-1c (SREBP-1c) gene in the liver. Induction of the hepatic expression of Srebp1c and of its target genes involved in fatty acid/triglyceride synthesis by fasting and refeeding or by hepatic expression of an active form of PI3K was inhibited in L- $\lambda \mathrm{KO}$ mice compared with that in control animals. Expression of Srebp1c induced by insulin or by active PI3K in primary cultured rat hepatocytes was inhibited by a dominant-negative form of PKC $\lambda$ and was mimicked by overexpression of WT PKC $\lambda$. Restoration of PKC $\lambda$ expression in the liver of L- $\lambda$ KO mice with the use of adenovirus-mediated gene transfer corrected the metabolic abnormalities of these animals. Hepatic PKC $\lambda$ is thus a determinant of hepatic lipid content and whole-body insulin sensitivity.
\end{abstract}

J. Clin. Invest. 112:935-944 (2003). doi:10.1172/JCI200318816.

\section{Introduction}

The liver is essential for both carbohydrate and lipid homeostasis. Individuals with type 2 diabetes often exhibit impairment of insulin action in the liver (1), and liver-specific inhibition of insulin signaling in mice results in glucose intolerance and dyslipidemia (2), indicating the physiological importance of hepatic insulin action in energy homeostasis. Among the signaling molecules activated by insulin, PI3K plays a key role in the metabolic actions of this hormone $(3,4)$. Prevention of the insulin-induced activation of PI3K in the liver of mice thus results in glucose intolerance and dyslipidemia (5).

Received for publication May 2, 2003, and accepted in revised form July 15, 2003.

Address correspondence to: Masato Kasuga, Department of Clinical Molecular Medicine, Division of Diabetes and Digestive and Kidney Diseases, Kobe University Graduate School of

Medicine, 7-5-1 Kusunoki-cho, Chuo-ku, Kobe 650-0017, Japan. Phone: 81-78-382-5861; Fax: 81-78-382-2080;

E-mail: kasuga@med.kobe-u.ac.jp.

Conflict of interest: The authors have declared that no conflict of interets exists.

Nonstandard abbreviations used: atypical PKC (aPKC); sterol regulatory element-binding protein-1c (SREBP-1c); glucose-6phosphatase catalytic subunit (G6PC); insulin receptor substrate (IRS); glucokinase (GCK); I- $\mathrm{\kappa B}$ kinase- $\beta$ (IKK $\beta$ ).
However, the signaling molecules that function downstream of PI3K to mediate the metabolic actions of insulin in living animals have remained unclear.

Two atypical PKC (aPKC) isozymes, PKC $\lambda$ and PKC $\xi$ (6), have been identified in mammals. We have previously shown that PKC $\lambda$ acts downstream of PI3K in PDGF or insulin signaling in cultured cells $(7,8)$. Furthermore, evidence suggests that 3 '-phosphoinositide-dependent kinase-1 (PDK-1), thought to be a key mediator of PI3K signaling, contributes to the activation of AGC protein kinases (which include Akt, p70 S6 kinase, p90 ${ }^{\text {rsk }}$, and aPKC) by phosphorylating these enzymes on residues located in their activation loops $(9,10)$. On the basis of these various observations, we hypothesized that PKC $\lambda$ participates in insulin action in vivo. To verify this hypothesis, using homologous recombination, we generated mice that harbor a PKC $\lambda$ gene containing loxP sites. Disruption of the corresponding aPKC genes in Caenorbabditis elegans and Drosophila melanogaster resulted in embryonic death $(11,12)$. As expected, PKC $\lambda$-deficient mice generated from the floxed PKC $\lambda$ transgenic animals also died during embryonic development (K. Akimoto et al., unpublished observations).

Given that the liver is one of the most important target organs of insulin, we next generated mice in which 
the floxed PKC $\lambda$ gene was specifically deleted in the liver as a result of Cre recombinase expression in this organ. Characterization of these animals has now revealed that $\mathrm{PKC} \lambda$ mediates the regulatory effect of insulin on hepatic triglyceride content by contributing to the expression of the gene for sterol regulatory element-binding protein-1c (SREBP-1c), and that the lack of PKC $\lambda$ in the liver results in increased insulin sensitivity.

\section{Methods}

Animals. Mice harboring a floxed PKC $\lambda$ gene in which exon 5 was flanked by loxP sequences $\left(P K C \lambda^{l o x /+}\right.$ mice $)$ were generated by homologous recombination (K. Akimoto et al., unpublished observations). Mice that express Cre recombinase under the control of the albumin gene promoter (Alb-Cre mice) (13) were provided by D. LeRoith (Diabetes Branch, National Institute of Diabetes and Digestive and Kidney Diseases, NIH, Bethesda, Maryland, USA). We used only male mice for the present studies. For the fasting-refeeding experiments, mice in the fasted group were deprived of food for 24 hours; mice in the refed group were deprived of food for 24 hours and then allowed access to food for 12 hours before analysis. The liver $\mathrm{X}$ receptor agonist T0901317 (kindly provided by K. Murakami, Kyorin Pharmaceutical Co., Tokyo, Japan) was administered daily by oral gavage at a dose of $10 \mathrm{mg} / \mathrm{kg}$ body mass for 4 days. For oral glucose intake experiments, mice deprived of food for 16 hours were loaded orally with glucose $(2 \mathrm{~g} / \mathrm{kg}$ body mass). For in vivo adenovirus-mediated gene transfer experiments, mice were injected with the indicated adenovirus vector $\left(1 \times 10^{8}\right.$ PFU) via the tail vein 72 hours before experiments.

Analysis of metabolic parameters. Blood glucose and plasma insulin concentrations were determined as described (5). For glucose tolerance and insulin tolerance tests, mice deprived of food for 16 hours were injected intraperitoneally with glucose $(2 \mathrm{~g} / \mathrm{kg}$ body mass); mice in the randomly fed state were injected intraperitoneally with human regular insulin $(0.75$ $\mathrm{U} / \mathrm{kg}$ ). Serum leptin and adiponectin concentrations were measured with a mouse leptin ELISA kit (Morinaga Institute of Biological Science, Yokohama, Japan) and a mouse/rat adiponectin ELISA kit (Otsuka Pharmaceutical Co. Ltd., Tokyo, Japan), respectively. Serum cholesterol, triglyceride, and FFA concentrations were determined with a cholesterol C-II kit, a triglyceride G kit, and a NEFA C kit, respectively (Wako Pure Chemical Industries Ltd., Osaka, Japan). For assay of the cholesterol and triglyceride contents of liver or hind limb skeletal muscle, lipids were extracted from the tissue as described (14) and the concentrations of the analytes in the extract were determined with a cholesterol C-II kit or a triglyceride G kit.

Northern blot, immunoblot, kinase activity, real-time quantitative RT-PCR analyses, and primary culture of hepatocytes. Total RNA $(\sim 15 \mu \mathrm{g})$ was subjected to Northern blot analysis essentially as described (15); autoradiograms were visualized and signal intensity was quantitated with a BAS2000 image analyzer (Fujifilm Co., Tokyo, Japan). The probes for the genes encoding PPAR- $\alpha$, acyl-CoA oxidase-1, and uncoupling protein- 2 mRNA's (mouse full-length cDNAs) were synthesized by PCR; the other probes were as described $(5,16,17)$. Primary cultures of rat hepatocytes were prepared and subjected to adenovirus infection as described (15).

For assay of the expression of the genes encoding SREBP-1 and fatty acid synthase, cells infected or not with adenoviruses were incubated for 6 hours and 20 hours, respectively, with $100 \mathrm{nM}$ insulin or with $10 \mu \mathrm{M}$ T0901317; for assay of the expression of the genes encoding phosphoenolpyruvate carboxykinase-1 (PKC-1) and glucose-6-phosphatase catalytic subunit (G6PC), cells were incubated for 6 hours with $500 \mathrm{nM}$ dexamethasone and $0.1 \mathrm{mM}$ PCPT-cAMP in the absence or presence of $100 \mathrm{nM}$ insulin. Adenovirus vectors encoding a dominant-negative mutant of PKC $\lambda$ (AxCA $\lambda$ KD), WT PKC $\lambda$ (AxCA $\lambda$ WT) (8), or a Myc epitope-tagged active form of PI3K (the $110-\mathrm{kDa}$ catalytic subunit fused with a myristoylation signal sequence at its $\mathrm{NH}_{2}$-terminus; AxCAMyr-p110) (15, 18) were described previously, and that encoding $\beta$-gal (AxCALacZ) was kindly provided by I. Saito (University of Tokyo, Tokyo, Japan). The antibodies specific for mouse SREBP-1c were as described (14).

For assay of the abundance of $\mathrm{PKC} \lambda$ and $\mathrm{PKC} \zeta$, total tissue homogenates were subjected to immunoprecipitation with antibodies to PKC $\lambda(\alpha \lambda 190)$ or to PKC $\zeta(\alpha \zeta 170)(8)$, and the resulting precipitates were subjected to immunoblot analysis with antibodies to PKC $\lambda / \mathrm{l}$ (Transduction Laboratories, Lexington, Kentucky, USA) or to PKCऽ (Life Technologies Inc., Rockville, Maryland, USA), respectively. Antibodies that recognize both $\mathrm{PKC} \lambda$ and PKC' (C-20, a rabbit polyclonal antibody, and C-20-G, a goat polyclonal antibody) were obtained from Santa Cruz Biotechnology (Santa Cruz, California, USA). For immunodepletion of aPKC, liver homogenates were subjected to three sequential immunoprecipitations for 120 minutes with $\alpha \lambda 190$ or with $\alpha \xi 170$. The supernatants of the immunoprecipitations were then subjected to immunoprecipitation with C-20-G and the precipitates were subjected to immunoblot analysis with $\mathrm{C}-20$. The kinase activity of aPKC was assayed in the immunoprecipitates with $\alpha \lambda 190$ or with $\alpha \zeta 170$ as described previously (8). For real-time quantitative reverse transcription and PCR analysis, cDNA synthesized from total RNA was evaluated in a sequence detector (model 7900; Applied Biosystems, Foster City, California, USA) with specific primers and SYBR Green PCR Master Mix (Applied Biosystems). The relative abundance of mRNA's was calculated with $36 B 4$ mRNA as the invariant control. The primers used were as follows: mouse Srebp1a, 5'-GGAACAGACACTGGCCGAGA-3' (sense) and $5^{\prime}$-GCATAGGGGGCGTCA-3' (antisense); mouse Srebp1c, 5'-ATCGGCGCGGAAGCTGTCGGGGTAGCGTC-3' (sense) and 5'-ACTGTCTTGGTTGTTGATGAGCTGGAGCAT- $3^{\prime}$ (antisense). The primers for mouse 36B4 were as described previously (5). 


\section{Results}

Generation of mice with liver-specific deficiency of $P K C \lambda$. We bred $P K C \lambda l^{o x /+}$ mice with Alb-Cre mice (13). We then bred the $P K C \lambda^{l o x /+}$, Alb-Cre offspring of this cross with $P K C \lambda^{l o x /+}$ mice. The offspring of this breeding were born in a Mendelian ratio $\left(P K C \lambda^{+/+}, n=12\right.$, or $9.9 \%$; $P K C \lambda^{+/+}$, Alb-Cre, $n=14$, or $11.6 \% ; P K C \lambda^{\text {lox } /+}, n=29$, or $24.0 \%$; $P K C \lambda^{l o x /+}$, Alb-Cre, $n=33$, or $27.3 \%$; $P K C \lambda^{l o x} /$ lox , $n=15$, or $12.4 \%$; PKC $\lambda^{l o x} /$ lox , Alb-Cre, $n=18$, or $\left.14.9 \%\right)$.

Two closely related isoforms comprise aPKC: PKC $\lambda$ and PKC $\zeta$ (6). We first investigated the amounts of $\mathrm{PKC} \lambda$ and $\mathrm{PKC} \zeta$ in liver homogenates of $P K C \lambda^{+/+}$(WT) mice. After three sequential immunoprecipitations with antibodies to $\mathrm{PKC} \lambda$ or to $\mathrm{PKC} \zeta$, the corresponding isoforms of aPKC were almost completely depleted from the homogenates (Figure 1a). aPKC protein, detected by antibodies that recognize both PKC $\lambda$ and PKC $\zeta$, was present both in the PKC $\lambda$-depleted and the PKC $\zeta$-depleted homogenates, suggesting that liver contains both PKC $\lambda$ and PKC $\zeta$. The relative abundance of each isoform was comparable. The amount of PKC $\lambda$ in the liver of $P K C \lambda^{\text {lox/lox }}$, Alb-Cre $(\mathrm{L}-\lambda \mathrm{KO})$ mice was markedly reduced compared with that in the liver of WT, $P K C \lambda^{+/+}$, Alb-Cre (WT-Cre), and PKC $\lambda^{l o x} /$ lox $\left(\lambda^{l o x} / l o x\right)$ animals (Figure $1 \mathrm{~b}$ ). In contrast, the hepatic abundance of PKC $\zeta$ was similar among all four genotypes of mice.

Given the similarity in the hepatic expression level of $\mathrm{PKC} \lambda$ in WT, WT-Cre, and $\lambda^{l o x / l o x}$ mice, which indicates that neither the insertion of the loxP sequences in the $\mathrm{PKC} \lambda$ gene nor the expression of Cre recombinase alone affected the abundance of PKC $\lambda$, we performed subsequent experiments with $\mathrm{L}-\lambda \mathrm{KO}$ and $\lambda^{\text {lox/lox }}$ mice. The kinase activity of $\mathrm{PKC} \lambda$, but not that of $\mathrm{PKC} \zeta$, was greatly reduced in the liver of $\mathrm{L}-\lambda \mathrm{KO}$ mice compared with that in the liver of $\lambda^{l o x} / l o x$ mice (Figure $1 \mathrm{c}$ ). The abundance of PKC $\lambda$ in other tissues, including skeletal muscle, adipose tissue, the pancreas, the lung, and the kidney, did not differ between L- $\lambda \mathrm{KO}$ and $\lambda^{\text {lox/lox }}$ mice (Figure 1d). The abundance of PKC $\zeta$ in the two genotypes of mice was similar in all tissues we examined. The gross appearance and histology of the liver, including the arrangement of hepatocytes in plates and the structure of hepatic lobules, appeared normal in $\mathrm{L}-\lambda \mathrm{KO}$ mice (data not shown). The serum levels of aspartate aminotransferase, alanine aminotransferase, alkaline phosphatase, lactate dehydrogenase, and albumin also did not differ significantly between L- $\lambda \mathrm{KO}$ and $\lambda^{l o x / l o x}$ mice (data not shown). These results indicate that the lack of PKC $\lambda$ did not affect the development or general function of the liver.

Metabolic characteristics of $L-\lambda K O$ mice. The body mass, mass of the liver, and mass of epididymal fat tissue of $\mathrm{L}-\lambda \mathrm{KO}$ mice were similar to those of $\lambda^{\text {lox } / l o x}$ mice (Table $1)$. The serum concentrations of triglyceride, cholesterol, FFAs, leptin, and adiponectin in the randomly fed state also did not differ between mice of the two genotypes. Although the blood glucose concentration in the randomly fed state was similar in the two types of mice, the plasma concentration of insulin in $\mathrm{L}-\lambda \mathrm{KO}$ mice was significantly lower than that in $\lambda^{l o x} / l^{\circ x}$ mice. Blood glucose concentrations during a glucose tolerance test were similar in both $\mathrm{L}-\lambda \mathrm{KO}$ and $\lambda^{\text {lox/lox }}$ mice (Figure $2 \mathrm{a}$ ). Again, however, the increase in plasma insulin concentration induced by glucose intake was smaller in $\mathrm{L}-\lambda \mathrm{KO}$ mice than in $\lambda^{\text {lox } / l o x}$ animals (Figure $2 \mathrm{~b}$ ). Moreover, the glucose-lowering effect of exogenously administered
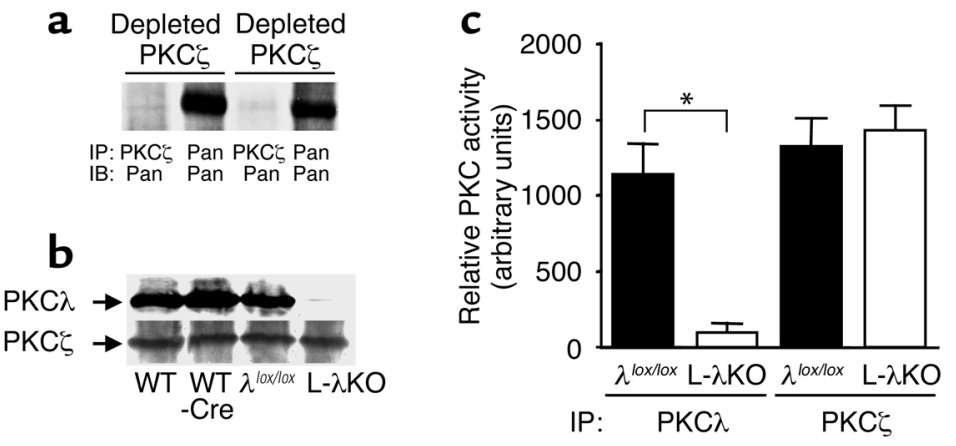

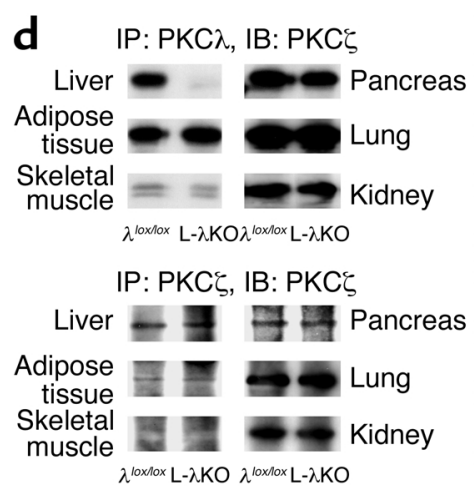

\section{Figure 1}

The abundance and kinase activity of PKC $\lambda$ and PKC $\zeta$ in mice with liver-specific PKC $\lambda$ deficiency. (a) Total homogenates prepared from the liver of 20 -week-old $P K C \lambda^{+/+}$mice were subjected to three sequential immunoprecipitations with antibodies to PKC $\lambda$ (depleted PKC $\lambda$ ) or to PKC $\zeta$ (deplete PKC $\zeta$ ). The resultant supernatants were subjected to immunoprecipitation with antibodies to PKC $\lambda$, to PKC $\zeta$, or with antibodies that recognize both PKC $\lambda$ and PKC (Pan), and the precipitates were subjected to immunoblot analysis with antibodies that recognize both PKC $\lambda$ and PKC $\zeta$ (Pan). (b) Total homogenates prepared from the liver of 18-week-old $P K C \lambda^{+/+}(\mathrm{WT}) ; P K C \lambda^{+/+}$, Alb-Cre (WT-Cre); $P K C \lambda^{\text {lox } / \text { lox }}\left(\lambda^{\text {lox } / \text { lox }}\right)$; or PKC $\lambda^{\text {lox } / \text { lox }}$, Alb-Cre $(\mathrm{L}-\lambda K \mathrm{KO})$ mice were subjected to immunoprecipitation with antibodies to PKC $\lambda$ or to PKC $\zeta$, and the resulting precipitates were subjected to immunoblot analysis with corresponding antibodies. (c) Total homogenates prepared from the liver of $\lambda^{\text {lox } / l o x}$ or L- $\lambda$ KO mice were subjected to immunoprecipitation (IP) with antibodies to PKC $\lambda$ or to PKC $\zeta$, and the resulting precipitates were assayed for kinase activity. (d) Extracts of the indicated tissues prepared from $\lambda^{\text {lox/lox }}$ or L- $\lambda$ KO mice were subjected to immunoprecipitation with antibodies to $\mathrm{PKC} \lambda$ or to $\mathrm{PKC} \zeta$, and the resulting precipitates were subjected to immunoblot analysis (IB) with corresponding antibodies. Data in $\mathbf{a}, \mathbf{b}$, and $\mathbf{d}$ are representative of at least three mice of each genotype; those in $\mathbf{c}$ are mean \pm SEM of values from six mice. ${ }^{*} P<0.01$ for the indicated comparison (ANOVA). 
Table 1

Phenotypic comparison of $\lambda^{\text {lox } / \text { lox }}$ and L- $\lambda$ KO mice

\begin{tabular}{lcc}
\hline & $\lambda^{\text {lox/lox }}$ & L- $\lambda$ KO \\
Parameter & & \\
18-week-old mice & $31.7 \pm 0.7$ & $31.8 \pm 0.5$ \\
Body mass $(\mathrm{g})$ & $1.52 \pm 0.03$ & $1.49 \pm 0.06$ \\
Liver mass $(\mathrm{g})$ & $0.31 \pm 0.03$ & $0.34 \pm 0.03$ \\
Epididymal fat mass $(\mathrm{g})$ & & \\
12-week-old mice & $111.3 \pm 4.2$ & $119.4 \pm 2.2$ \\
Blood glucose $(\mathrm{mg} / \mathrm{dl})$ & $518 \pm 38$ & $356 \pm 37^{A}$ \\
Plasma insulin $(\mathrm{pg} / \mathrm{ml})$ & $107.8 \pm 4.4$ & $112.0 \pm 4.3$ \\
Serum cholesterol $(\mathrm{mg} / \mathrm{dl})$ & $101.6 \pm 8.3$ & $107.4 \pm 5.9$ \\
Serum triglyceride $(\mathrm{mg} / \mathrm{dl})$ & $0.91 \pm 0.07$ & $0.95 \pm 0.05$ \\
Serum FFAs $(\mathrm{mEq} / \mathrm{l})$ & $1.34 \pm 0.26$ & $1.39 \pm 0.22$ \\
Serum leptin $(\mathrm{ng} / \mathrm{ml})$ & $13.9 \pm 0.3$ & $14.6 \pm 0.4$ \\
Serum adiponectin $(\mu \mathrm{g} / \mathrm{ml})$ &
\end{tabular}

Data are from male mice in the randomly fed state at the indicated ages. Values are mean \pm SEM of $15-27$ animals. ${ }^{A} P<0.05$ vs. $\lambda^{\text {lox/lox }}$ (Student's $t$ test).

insulin was exaggerated in L- $\lambda \mathrm{KO}$ mice (Figure $2 \mathrm{c}$ ). These observations indicated that the insulin sensitivity of L- $\lambda \mathrm{KO}$ mice was increased.

Altered hepatic gene expression in $L-\lambda K O$ mice. Early events of hepatic insulin signaling, including phosphorylation of insulin receptor substrate-1 (IRS-1), IRS-2, and Akt, did not differ between $\mathrm{L}-\lambda \mathrm{KO}$ and $\lambda^{l o x} / \mathrm{lox}$ mice after bolus injection of insulin (Figure $2 \mathrm{~d}$ ). We have previously shown that PKC $\lambda$ contributes to PI3K-dependent gene expression induced by growth factors (7). We therefore examined L- $\lambda \mathrm{KO}$ mice for the hepatic expression of genes that are regulated by insulin. The abundance of mRNA's for glucokinase (GCK), PKC-1, and G6PC in the liver of randomly fed animals was similar in $\mathrm{L}-\lambda \mathrm{KO}$ and $\lambda^{l o x / l o x}$ mice (Figure 2e). However, the amount of transcripts encoding SREBP-1, a transcription factor that regulates the expression of genes important in triglyceride synthesis $(19,20)$, as well as the amount of those encoding fatty acid synthase, the gene for which is regulated by SREBP-1 $(19,20)$, were reduced by approximately $50 \%$ in the liver of $\mathrm{L}-\lambda \mathrm{KO}$ mice.

The expression of Srebp 1 and its target genes in the liver is induced when mice are refed after starvation $(21,22)$, a treatment that also results in an increase in the circulating insulin concentration. The increases in the hepatic expression of Srebp1, Fas, and the gene for stearoyl-CoA desaturase-1 (SCD-1; another target of SREBP-1) (19) induced by refeeding were inhibited by about $50 \%, 35 \%$, and $25 \%$, respectively, in L- $\lambda$ KO mice (Figure $2 \mathrm{f}$ ). Of the two splice variants of Srebp1 mRNA (19), only the abundance of Srebp1c mRNA, not that of Srebp1a mRNA, is increased in the liver in response to insulin or refeeding (22). The induction of Srebp1c expression in the liver in response to refeeding was inhibited by about $50 \%$ in $\mathrm{L}-\lambda \mathrm{KO}$ mice (Figure $2 \mathrm{~g}$ ). Moreover, immunoblot analysis with antibodies specific for SREBP-1c (14) revealed that the increase in the amount of this protein in a nuclear fraction of the liver induced by refeeding was markedly reduced in L- $\lambda \mathrm{KO}$ mice (Figure $2 \mathrm{~h}$ ).
We and other investigators have shown that the effect of insulin on the expression of Srebp 1 is mediated by a PI3K-dependent pathway $(15,23)$. We therefore next took advantage of the fact that systemic infusion of adenoviral vectors results in liver-specific expression of exogenous genes (5). Infusion of AxCAMyr-p110, but not of AxCALacZ, resulted in the expression of the Myr-p110 protein in the liver of both L- $\lambda \mathrm{KO}$ and $\lambda^{l o x} / / 0 x$ mice (Figure 2i); expression of Myr-p110 was not detected in skeletal muscle or adipose tissue (data not shown). Infusion of AxCAMyr-p110, but not of AxCALacZ, also resulted in a reduction in the blood glucose concentration of both L- $\lambda \mathrm{KO}$ and $\lambda^{l o x / l o x}$ mice in the fasted state, suggesting that activation of hepatic PI3K signaling lowers blood glucose concentration. However, the glucose-lowering effect of AxCAMyrp110 was greater in L- $\lambda$ KO mice than in the control animals, consistent with our observation that the glucose-lowering effect of exogenously administered insulin was exaggerated in $\mathrm{L}-\lambda \mathrm{KO}$ mice.

Although the hepatic expression of Srebp1 and Fas was induced by the infusion of AxCAMyr-p110 in both $\mathrm{L}-\lambda \mathrm{KO}$ and $\lambda^{l o x} /$ lox mice (Figure $2 \mathrm{j}$ ), the extent of this effect was greatly reduced in the former animals, indicating that PKC $\lambda$ functions as a downstream effector of PI3K in the induction of Srebp1c expression in the liver. Activation of the liver $\mathrm{X}$ receptor also increases the expression of Srebp1c in the liver $(16,24)$. The hepatic expression of Srebp 1 and Fas induced by gavage with T0901317, a liver X receptor agonist (24), was similar in $\mathrm{L}-\lambda \mathrm{KO}$ and $\lambda^{l o x} /$ lox mice (Figure $2 \mathrm{k}$ ), suggesting that the lack of PKC $\lambda$ does not affect the general machinery responsible for the induction of Srebp1c but rather results in a specific disruption in PI3K-dependent signaling that leads to increased expression of this gene.

Hepatic lipid content and expression of genes important in $\beta$-oxidation in $L-\lambda K O$ mice. The triglyceride content of the liver was reduced (Figure 3a), whereas the hepatic cholesterol content (Figure 3b) and the triglyceride content of skeletal muscle (Figure 3c) were unchanged in L- $\lambda \mathrm{KO}$ mice in the randomly fed state compared with the corresponding values for $\lambda^{l o x} /$ lox mice. The synthesis and breakdown ( $\beta$-oxidation) of triglyceride are the two major determinants of hepatic triglyceride content. The expression of genes that contribute to the oxidation of triglyceride in the liver, including those encoding PPAR- $\alpha$, acyl-CoA oxidase- 1 , and uncoupling protein-2, did not differ between $\mathrm{L}-\lambda \mathrm{KO}$ and $\lambda^{\operatorname{lox} / 0 x}$ mice in the randomly fed state (Figure 3d), suggesting that the reduced hepatic triglyceride content of $\mathrm{L}-\lambda \mathrm{KO}$ mice is attributable to the reduced expression of Srebp1c.

Hepatic accumulation of glycogen and the induction of Pck1 and G6pc by starvation/refeeding in L- $\lambda K O$ mice. The hepatic glycogen content in the randomly fed state (data not shown) and the increase in hepatic glycogen content in response to oral glucose intake (Figure 3e) were similar in $\mathrm{L}-\lambda \mathrm{KO}$ and $\lambda^{10 x / l o x}$ mice. The expression of Pck1 and G6pc in the liver is inhibited by refeeding after food deprivation. The effect of refeeding on the 
a

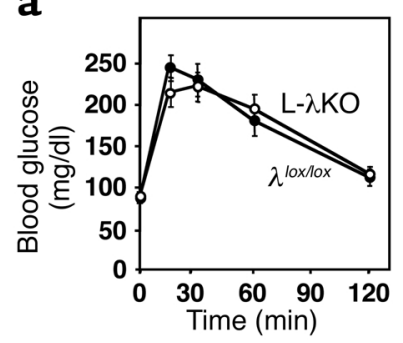

b

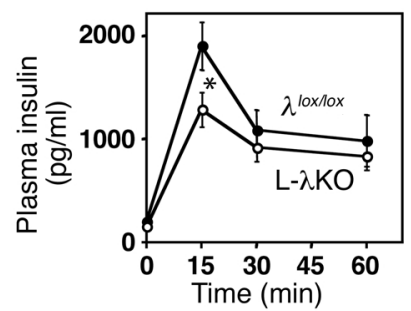

C

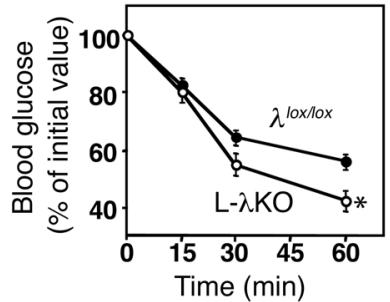

d

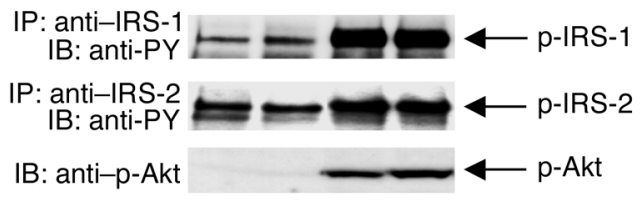

f

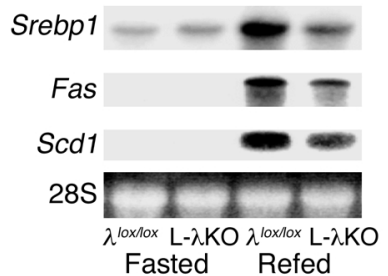

i
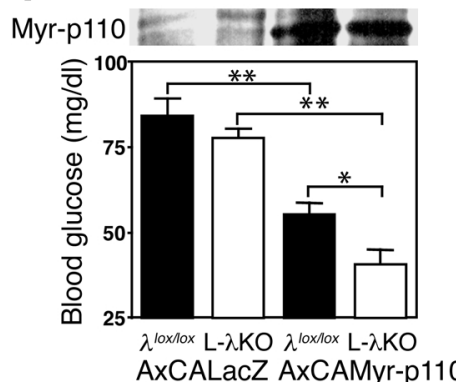

j $g_{1}$

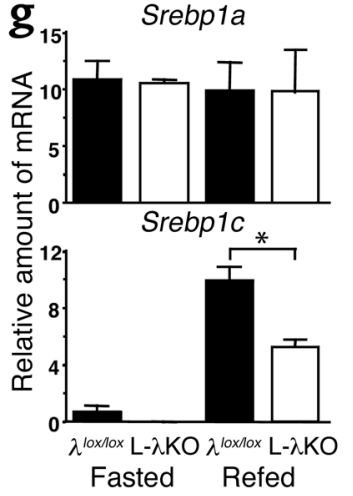

e

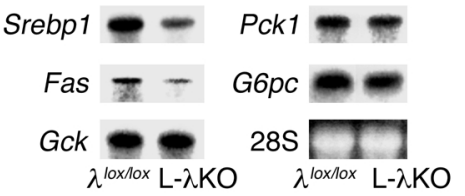

h

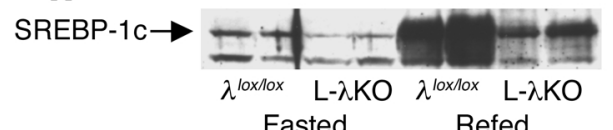

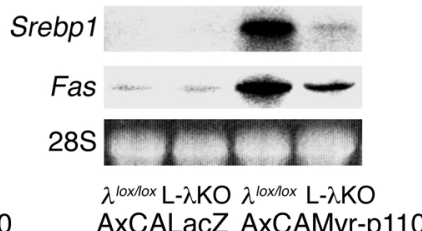

k

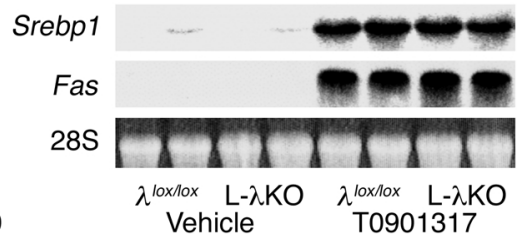

\section{Figure 2}

Glucose and insulin tolerance, insulin signaling, and hepatic gene expression in mice with liver-specific deficiency of PKC $\lambda$. (a-c) Blood glucose (a) and plasma insulin (b) concentrations during a glucose-tolerance test in L- $\lambda$ KO and $\lambda^{\text {lox } / \text { lox }}$ mice at 14 weeks of age, and blood glucose concentration during an insulin tolerance test at 12 weeks of age (c). Data are mean \pm SEM of values from nine to 20 mice. ${ }^{*} P<0.05$ vs. the corresponding value for $\lambda^{10 x / 10 x}$ mice (Student's $t$ test). (d) Tyrosine phosphorylation of IRS- 1 and IRS-2 and serine phosphorylation of Akt in the liver of $\lambda^{10 x / l o x}$ or L- $\lambda$ KO mice induced by a bolus injection of insulin. Liver homogenates prepared 2 minutes after administration of insulin ( $5 \mathrm{U} / \mathrm{kg}$ of body mass) or saline were subjected to immunoprecipitation with antibodies to IRS-1 or to IRS-2, and the resulting precipitates were subjected to immunoblot analysis with antibodies to phosphotyrosine (PY). Alternatively, liver homogenates were subjected directly to immunoblot analysis with antibodies specific for phosphorylated Akt (p-Akt). Data are representative of six mice of each genotype. (e-g) Total RNA extracted from the liver of $\lambda^{\text {lox/lox }}$ or L- $\lambda$ KO mice (18 weeks of age) in the randomly fed state $(n=8)(\mathbf{e})$ or after fasting with or without refeeding $(n=4-7)(\mathbf{f}$ and $\mathbf{g})$ was either separately combined and subjected to Northern blot analysis (e and $\mathbf{f}$ ) or subjected individually to RT-PCR analysis ( $\mathbf{g}$ ) for the indicated mRNA's. Ethidium bromide staining of $28 \mathrm{~S}$ rRNA is also shown for Northern analysis. ${ }^{*} P<0.01$ (ANOVA). (h) The nuclear fraction of liver homogenates prepared from $\lambda^{10 x / 10 x}$ or L- $\lambda$ KO mice after fasting with or without refeeding was subjected to immunoblot analysis with antibodies to SREBP-1c. Data shown are from two mice and are representative of four to six animals. (i and $\mathbf{j})$ Mice $\left(\lambda^{\text {lox/lox }}\right.$ or L- $\lambda$ KO) 16-18 weeks of age $(n=10-16)$ were injected with AxCAMyr-p110 or AxCALacZ and were subsequently deprived of food for 16 hours. The abundance of Myr-p110 in liver homogenates was then examined by immunoblot analysis with antibodies to Myc (i, upper panel), blood glucose concentration was determined (i, lower panel), and the amounts of Srebp1 and Fas mRNA's among separately combined total RNA extracted from the liver were evaluated by Northern analysis $(\mathbf{j})$. ${ }^{*} P<0.05,{ }^{*} P<0.01$ (ANOVA). (k) Total RNA extracted from the liver of $\lambda^{10 x / l o x}$ or L- $\lambda$ KO mice treated with either T0901317 or vehicle was separately combined and subjected to Northern blot analysis for Srebp1 and Fas mRNA's. Data are shown for two mice and are representative of four animals. 
hepatic expression of these genes was slightly exaggerated in $\mathrm{L}-\lambda \mathrm{KO}$ mice compared with that apparent in $\lambda^{l o x} /$ lox animals (Figure $3 \mathrm{f}$ ). These results thus suggested that PKC $\lambda$ signaling is not required for either the hepatic accumulation of glycogen or for inhibition of the expression of gluconeogenesis genes in the liver. The slight enhancement of refeeding-induced suppression of Pck1 and G6pc expression apparent in $\mathrm{L}-\lambda \mathrm{KO}$ mice may be related to the increased insulin sensitivity of these animals.

Effects of dominant-negative and WT PKC入 on insulininduced expression of Srebp1 in cultured hepatocytes. To confirm a causal relation between PKC $\lambda$ deficiency and the altered hepatic expression of Srebp 1 in L- $\lambda \mathrm{KO}$ mice, we examined the effect of PKC $\lambda$ signaling on the abundance of Srebp1 mRNA in primary cultures of rat hepatocytes. Incubation of the cells with insulin induced an increase in the amounts of Srebp1 and Fas mRNA's (Figure $4 \mathrm{a}$ ), and this effect was inhibited by adenovirusmediated expression of $\lambda \mathrm{KD}$, which acts in a dominant-negative manner $(7,8)$. Expression of $\lambda \mathrm{KD}$ also inhibited the increase in the amount of Srebp1 mRNA induced by Myr-p110 (Figure 4b). In contrast, $\lambda$ KD did not affect either the insulin-induced inhibition of Pck1 and G6pc expression (Figure 4c) or the expression of Srebp1 induced by T0901317 (Figure 4d), indicating that PKC $\lambda$ signaling specifically contributes to insulininduced expression of Srebp1. Moreover, expression of recombinant WT PKC $\lambda$ increased the abundance of Srebp1 and Fas mRNA's in the absence of insulin (Figure $4 \mathrm{e}$ ), indicating that $\mathrm{PKC} \lambda$ signaling is sufficient for the induction of these genes.

Restoration of PKC $\lambda$ expression reverses the decrease in hepatic lipid content and the increase in insulin sensitivity in L- $\lambda \mathrm{KO}$ mice. To verify that the altered insulin sensitivity and hepatic triglyceride content of L- $\lambda \mathrm{KO}$ mice are attributable to the lack of PKC $\lambda$ in the liver, we restored the hepatic expression of this enzyme in $\mathrm{L}-\lambda \mathrm{KO}$ animals. Infusion of $A x C A \lambda W T$ into $L-\lambda K O$ mice resulted in the expression of PKC $\lambda$ in the liver at a level similar to that apparent in $\lambda^{l o x / l o x}$ mice (Figure 5a). The restoration of PKC $\lambda$ expression in the liver increased both the abundance of Srebp 1 mRNA and the triglyceride content in this organ of $\mathrm{L}-\lambda \mathrm{KO}$ mice. Blood glucose concentration was similar in $\mathrm{L}-\lambda \mathrm{KO}$ mice infused with $\mathrm{AxCA} \lambda \mathrm{WT}$ or with AxCALacZ and in $\lambda^{\text {lox/lox }}$ mice infused with AxCALacZ or with PBS. The plasma insulin concentration of $\mathrm{L}-\lambda \mathrm{KO}$ mice was increased by infusion of AxCA $\lambda W T$, but not of AxCALacZ, to an extent similar to that apparent in $\lambda^{l o x / l o x}$ mice infused with AxCALacZ or with PBS (Figure 5b). Moreover, the enhancement of the glucose-lowering effect of exogenously administered insulin apparent in $\mathrm{L}-\lambda \mathrm{KO}$ mice was also reversed by restoration of PKC $\lambda$ expression in the liver (Figure 5c). These results thus indicate that the changes in the expression of Srebp1 and in triglyceride
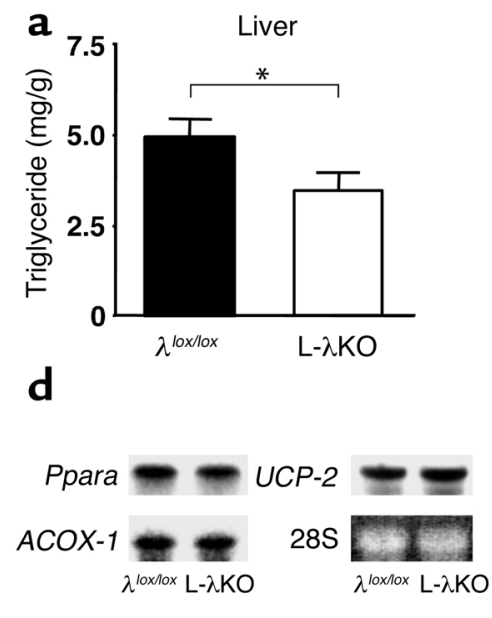

Figure 3

Hepatic lipid and glycogen content and the expression of genes involved in $\beta$-oxidation and gluconeogenesis in mice with liver-specific PKC $\lambda$ deficiency. ( $\mathbf{a}-\mathbf{c})$ Triglyceride (a) and cholesterol (b) content of the liver and triglyceride content of hind limb muscle (c) of $L-\lambda K O$ and $\lambda$ lox/lox mice in the randomly fed state at 18 weeks of age. Data are expressed as $\mathrm{mg}$ analyte/g wet tissue and are the mean \pm SEM from seven mice. ${ }^{*} P<0.05$ (Student's $t$ test). (d) Total RNA extracted from the liver of $\lambda^{\text {lox/lox }}$ or L- $\lambda$ KO mice $(n=8)$ at 18 weeks of age and in the randomly fed state was separately combined and subjected to Northern blot analysis for mRNA's encoding PPAR- $\alpha$, acyl-CoA oxidase-1 (ACOX-1), and uncoupling protein-2 (UCP-2). (e) Hepatic glycogen content of $\lambda^{\text {lox } / \text { lox }}$ or L- $\lambda$ KO mice at 20 weeks of age before (Pre) and 2 hours after (Post) oral glucose intake. Data are mean \pm SEM from four to six mice. (f) Total RNA extracted from the liver of $\lambda^{\text {lox/lox }}$ or L- $\lambda$ KO mice (18 weeks of age) after fasting with or without refeeding $(n=4-7)$ was separately combined and subjected to Northern blot analysis with probes specific for Pck1 or G6pc mRNA's. 
a
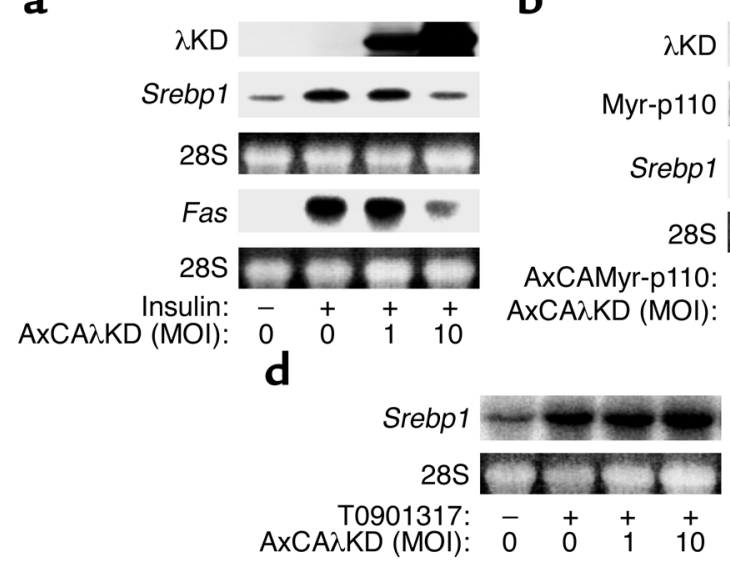

C

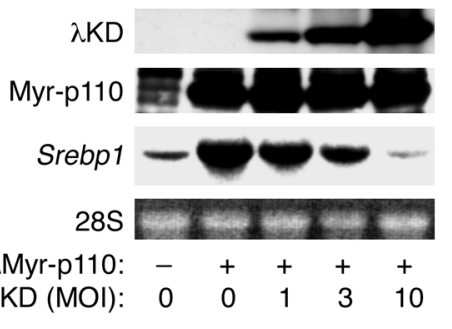

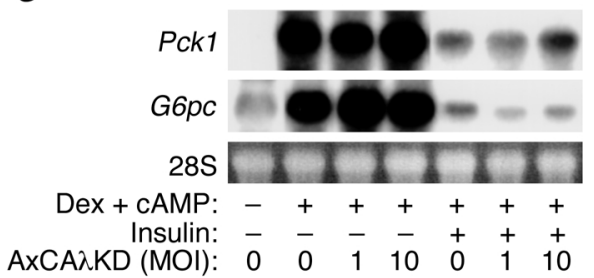

e

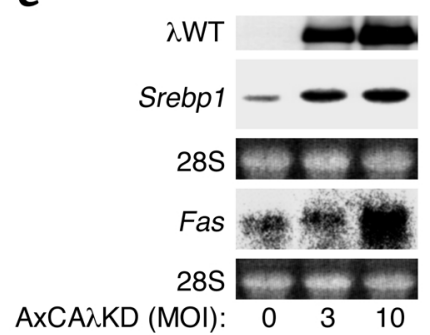

Figure 4

Effects of dominant-negative and WT PKC $\lambda$ on the expression of Srebp1, Fas, Pck1, and G6pc in primary cultured rat hepatocytes. (a, c, and d) Cells that had been infected (or not) with an adenoviral vector for a dominant-negative form of PKC $\lambda$ (AxCA $\lambda K D)$ at the indicated MOI (in PFU/cell) were incubated in the absence or presence of insulin, dexamethasone (Dex), or T0901317, plus pCPT-cAMP (these two agents were used to induce the expression of $P c k 1$ and $G 6 p c$ ), as indicated. Total cell lysates were then subjected to immunoblot analysis with antibodies to PKC $\lambda$, and total RNA extracted from the cells was subjected to Northern blot analysis with probes specific for Srebp 1, Fas, Pck1, or G6pc mRNA's. (b) Cells that had been infected (or not) with an adenovirus encoding a constitutively active form of PI3K (AxCAMyr-p110) at an $\mathrm{MOI}$ of three plaque-forming units/cell were then infected with AxCA $\lambda K D$ at the indicated MOI. Total cell lysates were subjected to immunoblot analysis with antibodies to PKC $\lambda$ or to Myc (for the detection of Myr-p110), and total RNA extracted from the cells was subjected to Northern blot analysis with a probe specific for Srebp1 mRNA. (e) Cells were infected (or not) with an adenovirus encoding WT PKC $\lambda(A x C A \lambda W T)$ at the indicated $\mathrm{MOI}$, after which cell lysates were subjected to immunoblot analysis with antibodies to PKC $\lambda$ and total RNA extracted from the cells was subjected to Northern blot analysis with probes specific for Srebp 1 or Fas mRNAs. All data are representative of at least three independent experiments.

content in the liver as well as in whole-body insulin sensitivity apparent in $\mathrm{L}-\lambda \mathrm{KO}$ mice are directly attributable to the lack of PKC $\lambda$ in the liver.

\section{Discussion}

On the basis of observations with cultured cells $(7,8)$, we hypothesized that PKC $\lambda$ participates in insulin action in vivo as a downstream effector of PI3K. Our present results now demonstrate such a function for $\mathrm{PKC} \lambda$, at least in the liver. Several of the metabolic effects of insulin in the liver are exerted through the regulation of gene expression. We have previously shown that the regulation by insulin of the expression of Gck, Srebp1c, G6pc, and Pck1 in mouse liver is mediated by PI3K (5). Of these four genes, each of which participates in the metabolic actions of insulin, we have now revealed that the expression of Srebp1c, a key regulator of fatty acid and triglyceride synthesis (19, 20 ), is regulated by PKC $\lambda$ acting downstream of PI3K. At present, a signaling pathway that links PKC $\lambda$ and the expression of Srebp1c remains unclear. Cycloheximide, an inhibitor of general protein synthesis, has been shown to prevent insulin-induced expression of Srebp1c in cultured hepatocytes (25), suggesting that de novo protein synthesis is required for this action of insulin. PKC $\lambda$ thus may contribute to induce expres- sion of such a protein involved in the transcriptional activation of Srebp1c.

The hepatic expression of Srebp1c induced by refeeding or by an active PI3K was markedly, but not completely, prevented in L- $\lambda \mathrm{KO}$ mice, suggesting that the induction of Srebp1c is not solely dependent on PKC $\lambda$ signaling. Given that the liver expresses both PKC $\lambda$ and PKC $\zeta$, PKC $\zeta$ may be responsible for the residual signaling of Srebp1c. A membrane-targeted form of Akt that exhibits higher kinase activity than does WT Akt increases the abundance of Srebp1c mRNA when it is expressed in primary cultured hepatoyctes (23), suggesting that Akt, a downstream effector of PI3K, may also contribute to the induction of Srebp1c. However, we have previously shown that the inhibition of endogenous Akt activity with the use of a dominant-negative mutant of the kinase did not prevent, but rather augmented, insulin-induced expression of Srebp1c (15). Moreover, in $a b / o b$ mice and a mouse model with lipodystrophic diabetes, the abundance of Srebp1c mRNA is increased, whereas insulin-induced phosphorylation of Akt is markedly reduced in the liver of these animals (26). Mice lacking Akt2, a major isoform of Akt in the liver, have been established (27). The physiological importance of Akt in the induction of Srebp $1 \mathrm{c}$ in vivo may be revealed by characterization of the mutant mice. 
a

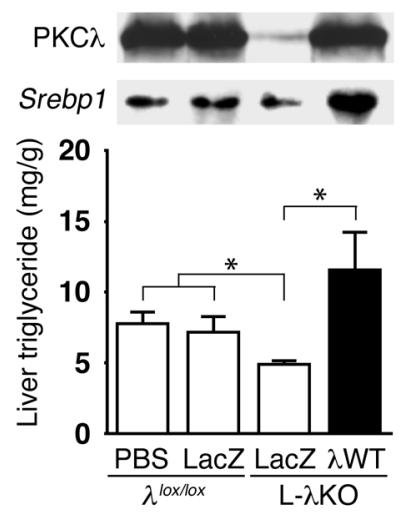

b

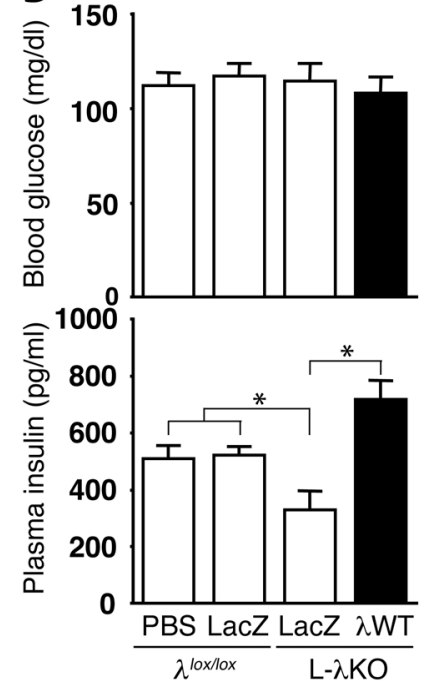

C

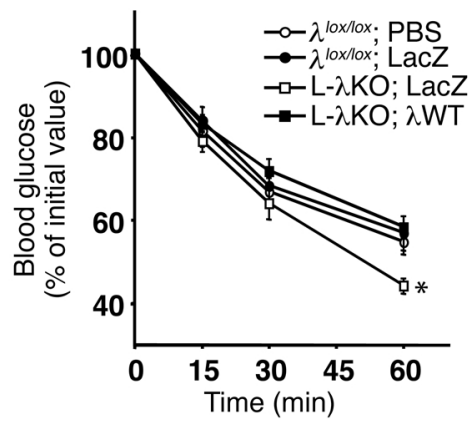

\section{Figure 5}

Effects of adenovirus-mediated restoration of PKC $\lambda$ expression in the liver of mice with liver-specific PKC $\lambda$ deficiency on hepatic lipid content, expression of Srebp 1 in the liver, and insulin sensitivity. (a) Twenty-week-old $\lambda^{\text {lox } / \text { lox }}$ or L- $\lambda$ KO mice $(n=7-9)$ were injected with PBS or with adenoviruses encoding either $\beta$-gal (LacZ) or WT PKC $\lambda(\lambda W T)$, as indicated. Total liver homogenates were subsequently subjected to immunoprecipitation and immunoblot analysis with antibodies to PKC $\lambda$ (upper panel; data are representative of three experiments). Total RNA extracted from the liver was separately combined and subjected to Northern blot analysis with a probe specific for Srebp 1 mRNA (middle panel), and hepatic triglyceride content was determined (lower panel; data are shown as mean \pm SEM). ${ }^{*} P<0.05$ (ANOVA). (b and $\mathbf{c}$ ) Twelve-week-old $\lambda^{\text {lox } / \text { lox }}$ or L- $\lambda$ KO mice were injected with PBS or with adenoviruses encoding either $\beta$-gal or WT PKC $\lambda$, after which blood glucose and plasma insulin concentrations were determined in the randomly fed state (b) or an insulin tolerance test was performed (c). Data represent mean \pm SEM from six to ten mice. ${ }^{*} P<0.05$ for the indicated comparisons $(\mathbf{b})$ or for the comparison of L- $\lambda$ KO mice injected with the adenovirus encoding $\beta$-gal vs. all other conditions.

Atypical PKC isozymes are evolutionarily conserved proteins required for the formation of apical-basal polarity in cells (28), which is important for the structural organization and function of organs. However, the structures of hepatic lobules and hepatocyte plates and the serum parameters of general liver function appeared normal in $\mathrm{L}-\lambda \mathrm{KO}$ mice. This observation may be attributable to the fact that disruption of the PKC $\lambda$ gene was accomplished by Cre recombinase expressed under the control of the promoter of the albumin gene, which is a marker gene of fully differentiated hepatocytes. A role for PKC $\lambda$ in the development of cellular polarity in the liver might be revealed by characterization of hepatectomy-induced liver regeneration in $\mathrm{L}-\lambda \mathrm{KO}$ mice, given that the liver regenerates predominantly through the replication of mature hepatocytes under this experimental condition (29).

An unexpected finding of the present study was that $L-\lambda K O$ mice exhibit increased insulin sensitivity. The tissue-specific disruption of a gene important for insulin signaling thus paradoxically resulted in an increase in whole-body insulin sensitivity. A similar phenomenon has been observed with mice lacking the insulin receptor specifically in adipose tissue (30). Restoration of the hepatic expression of PKC $\lambda$ reversed this metabolic phenotype of L- $\lambda$ KO mice, indicating that the lack of PKC $\lambda$ in the liver is indeed responsible for the increased insulin sensitivity of these animals. The precise mechanism that underlies this phenomenon remains unclear. Evidence suggests that the alteration of fatty acids/triglyceride metabolism in insulin's target tissues is an important determinant of insulin sensitivity. The increase in circulating FFAs leads to insulin resistance and the accumulation of triglyceride in skeletal muscle (31), and triglyceride content in the liver or in skeletal muscle negatively correlates with insulin sensitivity in humans $(32,33)$. Leptin and adiponectin, the two major fat-derived hormones, increase insulin sensitivity and concomitantly reduce hepatic triglyceride content (probably by promoting fatty acid oxidation) in an animal model of insulin resistance or in humans with lipodystrophic diabetes (34-36). Moreover, overexpression of lipoprotein lipase in liver or skeletal muscle resulted in an increase in fatty acid metabolites and consequently in the accumulation of triglyceride in the respective tissue, as well as insulin resistance (37). The decrease in the hepatic expression of the lipogenic genes and the subsequent alterations in fatty acid metabolism in L- $\lambda \mathrm{KO}$ mice may thus be related to the increased insulin sensitivity of these animals.

TNF- $\alpha$ secreted from adipose tissue is implicated in the development of obesity-induced insulin resistance (38). Atypical PKC is activated by cytokines, including TNF- $\alpha$ and IL-1 $(39,40)$, and directly phosphorylates and activates I- $\kappa B$ kinase- $\beta$ (IKK $\beta$ ) (41). Administration of salicylic acid derivatives that inhibit IKK $\beta$ (42) was shown to increase insulin sen- 
sitivity both in rodent models of diabetes and in human subjects $(43,44)$. Moreover, heterozygous disruption of the IKK $\beta$ gene ameliorated the insulin resistance of obese model mice (44). It is therefore possible that the hepatic deficiency of PKC $\lambda$ in $\mathrm{L}-\lambda \mathrm{KO}$ mice results in inhibition of a TNF- $\alpha / \mathrm{PKC} \lambda /$ IKK $\beta$ signaling pathway and a consequent increase in whole-body insulin sensitivity.

Transgenic mice that overexpress lipoprotein lipase in skeletal muscle or the liver exhibit an impairment of the insulin-induced increase in PI3K activity associated with IRS-1 or IRS-2, respectively (37). Moreover, administration of salicylic acid derivatives enhanced insulin-induced tyrosine phosphorylation of the insulin receptor in genetically obese animals (44). However, we did not detect changes in the insulininduced phosphorylation of IRS proteins or of Akt in the liver of L- $\lambda \mathrm{KO}$ mice. Although we cannot exclude the possibility that a small increase in the extent of insulin signaling went undetected under our experimental conditions, it is possible that the enhancement of insulin action apparent in L- $\lambda \mathrm{KO}$ mice occurs at a step other than IRS or Akt phosphorylation. Evidence suggests that $\mathrm{PKC} \zeta$ participates in a negative feedback pathway of insulin signaling leading to the phosphorylation of IRS proteins in cultured cells (45). Given that the insulin-induced phosphorylation of IRS proteins was not significantly increased in the liver of $\mathrm{L}-\lambda \mathrm{KO}$ mice, PKC $\lambda$ appears not to participate in such a negative feedback pathway in mouse liver.

In summary, we have shown that, among the various metabolic actions of insulin, PKC $\lambda$ specifically contributes to induction of the expression of Srebp1c and of its target genes important in triglyceride synthesis in the liver. Animal models of insulin resistance or obesity often manifest increases both in lipid content and in the expression of Srebp1c in the liver $(26,46)$. Reagents that block PKC $\lambda$ signaling specifically in the liver might thus prove effective for reducing hepatic Srebp1c expression and consequently hepatic triglyceride content, as well as for ameliorating insulin resistance.

\section{Acknowledgments}

We thank D. LeRoith, T. Noguchi, H. Nakajima, N. Iritani, D.K. Granner, and K. Murakami for Alb-Cre mice, probes for Gck, G6pc, Fas, and Pck1, and for T0901317, respectively. This work was supported by a grant from the Ministry of Education, Culture, Sports, Science, and Technology of Japan (to M. Kasuga and W. Ogawa), a grant-in-aid for the Research for the Future Program from the Japan Society for the Promotion of Science (to M. Kasuga), and a grant from Cooperative Link of Unique Science and Technology for Economy Revitalization (CLUSTER) (to M. Kasuga).

1. DeFronzo, R.A. 1997. Pathogenesis of type 2 diabetes: metabolic and molecular implications for identifying diabetic genes. Diabetes Rev. 5:177-269.

2. Michael, M.D., et al. 2000. Loss of insulin signaling in hepatocytes leads to severe insulin resistance and progressive hepatic dysfunction. Mol. Cell. 6:87-97.

3. Ogawa, W., Matozaki, T., and Kasuga, M. 1998. Role of binding proteins to IRS-1 in insulin signalling. Mol. Cell. Biochem. 182:13-22.

4. Shepherd, P.R., Withers, D.J., and Siddle, K. 1998. Phosphoinositide 3-kinase: the key switch mechanism in insulin signalling. Biochem. J. 333:471-490.

5. Miyake, K., et al. 2002. Hyperinsulinemia, glucose intolerance, and dyslipidemia induced by acute inhibition of phosphoinositide 3-kinase signaling in the liver. J. Clin. Invest. 110:1483-1491. doi:10.1172/JCI200215880.

6. Suzuki, A., Akimoto, K., and Ohno, S. 2003. Protein kinase C $\lambda / \mathrm{l}$ (PKC $\lambda / \mathrm{l})$ : a PKC isoform essential for the development of multicellular organisms. J. Biochem. (Tokyo). 133:9-16.

7. Akimoto, K., et al. 1996. EGF or PDGF receptors activate atypical PKC $\lambda$ through phosphatidylinositol 3-kinase. EMBOJ. 15:788-798.

8. Kotani, K., et al. 1998. Requirement of atypical protein kinase $\mathrm{C} \lambda$ for insulin stimulation of glucose uptake but not for Akt activation in 3T3-L1 adipocytes. Mol. Cell. Biol. 18:6971-6982.

9. Williams, M.R., et al. 2000. The role of 3-phosphoinositide-dependent protein kinase 1 in activating AGC kinases defined in embryonic stem cells. Curr. Biol. 10:439-448.

10. Le Good, J.A., et al. 1998. Protein kinase C isotypes controlled by phosphoinositide 3-kinase through the protein kinase PDK1. Science. 281:2042-2045.

11. Tabuse, Y. 1998. Atypical protein kinase C cooperates with PAR-3 to establish embryonic polarity in Caenorhabditis elegans. Development. 125:3607-3614.

12. Wodarz, A., Ramrath, A., Grimm, A., and Knust, E. 2000. Drosophila atypical protein kinase $\mathrm{C}$ associates with Bazooka and controls polarity of epithelia and neuroblasts. J. Cell Biol. 150:1361-1374.

13. Yakar, S., et al. 1999. Normal growth and development in the absence of hepatic insulin-like growth factor I. Proc. Natl. Acad. Sci. U. S. A. 96:7324-7329.

14. Yahagi, N., et al. 1999. A crucial role of sterol regulatory element-binding protein-1 in the regulation of lipogenic gene expression by polyunsaturated fatty acids. J. Biol. Chem. 274:35840-35844.

15. Matsumoto, M., et al. 2002. Role of the insulin receptor substrate 1 and phosphatidylinositol 3-kinase signaling pathway in insulin-induced expression of sterol regulatory element binding protein $1 \mathrm{c}$ and glucokinase genes in rat hepatocytes. Diabetes. 51:1672-1680.

16. Repa, J.J., et al. 2000. Regulation of mouse sterol regulatory elementbinding protein-1c gene (SREBP-1c) by oxysterol receptors, LXR $\alpha$ and LXR $\beta$. Genes Dev. 14:2819-2830.

17. Katsurada, A., et al. 1990. Effects of nutrients and hormones on transcriptional and post-transcriptional regulation of fatty acid synthase in rat liver. Eur. J. Biochem. 190:427-433.

18. Kitamura, T., et al. 1999. Insulin-induced phosphorylation and activation of cyclic nucleotide phosphodiesterase $3 \mathrm{~B}$ by the serine-threonine kinase Akt. Mol. Cell. Biol. 19:6286-6296.

19. Horton, J.D., Goldstein, J.L., and Brown, M.S. 2002. SREBPs: activators of the complete program of cholesterol and fatty acid synthesis in the liver. J. Clin. Invest. 109:1125-1131. doi:10.1172/JCI200215593.

20. Shimano, H., et al. 1999. Sterol regulatory element-binding protein-1 as a key transcription factor for nutritional induction of lipogenic enzyme genes. J. Biol. Chem. 274:35832-35839.

21. Horton, J.D., Bashmakov, Y., Shimomura, I., and Shimano, H. 1998. Regulation of sterol regulatory element binding proteins in livers of fasted and refed mice. Proc. Natl. Acad. Sci. U. S. A. 95:5987-5992.

22. Shimomura, I., et al. 1999. Insulin selectively increases SREBP-1c mRNA in the livers of rats with streptozotocin-induced diabetes. Proc. Natl. Acad. Sci. U. S. A. 96:13656-13661.

23. Fleischmann, M., and Iynedjian, P.B. 2000. Regulation of sterol regulatory-element binding protein 1 gene expression in liver: role of insulin and protein kinase B/cAkt. Biochem. J. 349:13-17.

24. Schultz, J.R., et al. 2000. Role of LXRs in control of lipogenesis. Genes Dev. 14:2831-2838.

25. Foretz, M., et al. 1999. ADD1/SREBP-1c is required in the activation of hepatic lipogenic gene expression by glucose. Mol. Cell. Biol. 19:3760-3768.

26. Shimomura, I., et al. 2000. Decreased IRS-2 and increased SREBP-1c lead to mixed insulin resistance and sensitivity in livers of lipodystrophic and ob/ob mice. Mol. Cell. 6:77-86.

27. Cho, H., et al. 2001. Insulin resistance and a diabetes mellitus-like syndrome in mice lacking the protein kinase Akt2 (PKB $\beta)$. Science. 292:1728-1731.

28. Ohno, S. 2001. Intercellular junctions and cellular polarity: the PARaPKC complex, a conserved core cassette playing fundamental roles in cell polarity. Curr. Opin. Cell Biol. 13:641-648.

29. Fausto, N., and Campbell, J.S. 2003. The role of hepatocytes and oval cells in liver regeneration and repopulation. Mech. Dev. 120:117-130.

30. Bluher, M., et al. 2002. Adipose tissue selective insulin receptor knockout protects against obesity and obesity-related glucose intolerance. Dev. Cell. 3:325-338. 
31. Bachmann, O.P., et al. 2001. Effects of intravenous and dietary lipid challenge on intramyocellular lipid content and the relation with insulin sensitivity in humans. Diabetes. 50:2579-2584.

32. Ryysy, L., et al. 2000. Hepatic fat content and insulin action on free fatty acids and glucose metabolism rather than insulin absorption are associated with insulin requirements during insulin therapy in type 2 diabetic patients. Diabetes. 49:749-758.

33. Krssak, M., et al. 1999. Intramyocellular lipid concentrations are correlated with insulin sensitivity in humans: a $1 \mathrm{H}$ NMR spectroscopy study. Diabetologia. 42:113-116.

34. Kakuma, T., et al. 2000. Leptin, troglitazone, and the expression of sterol regulatory element binding proteins in liver and pancreatic islets. Proc. Natl. Acad. Sci. U. S. A. 97:8536-8541.

35. Yamauchi, T., et al. 2001. The fat-derived hormone adiponectin reverses insulin resistance associated with both lipoatrophy and obesity. Nat. Med. 7:941-946.

36. Petersen, K.F., et al. 2002. Leptin reverses insulin resistance and hepatic steatosis in patients with severe lipodystrophy. J. Clin. Invest. 109:1345-1350. doi:10.1172/JCI200215001.

37. Kim, J.K., et al. 2001. Tissue-specific overexpression of lipoprotein lipase causes tissue-specific insulin resistance. Proc. Natl. Acad. Sci. U. S. A. 98:7522-7527.

38. Uysal, K.T., Wiesbrock, S.M., Mario, M.W., and Hotamisligil, G.S. 1997. Protection from obesity-induced insulin resistance in mice lacking TNF- $\alpha$ function. Nature. 389:610-614.
39. Muller, G., et al. 1995. PKC $\zeta$ is a molecular switch in signal transduction of TNF- $\alpha$, bifunctionally regulated by ceramide and arachidonic acid. EMBO J. 14:1961-1969.

40. Limatola, C., Barabino, C., Nista, A., and Santoni, A. 1997. Interleukin $1-\beta$-induced protein kinase $C-\zeta$ activation is mimicked by exogenous phospholipase D. Biochem. J. 321:497-501.

41. Lallena, M.J, Diaz-Meco, M.T., Bren, G., Paya, C.V., and Moscat, J. 1999. Activation of IкB kinase $\beta$ by protein kinase C isoforms. Mol. Cell. Biol. 19:2180-2188.

42. Yin, M.J., Yamamoto, Y., and Gaynor, R.B. 1998. The anti-inflammatory agents aspirin and salicylate inhibit the activity of IкB kinase- $\beta$. Nature. 396:77-80.

43. Hundal, R.S., et al. 2002. Mechanism by which high-dose aspirin improves glucose metabolism in type 2 diabetes. J. Clin. Invest. 109:1321-1326. doi:10.1172/JCI200214955.

44. Yuan, M., et al. 2001. Reversal of obesity- and diet-induced insulin resistance with salicylates or targeted disruption of Ikkß. Science. 293:1673-1677.

45. Liu, Y.F., et al. 2001. Insulin stimulates PKC $\zeta$-mediated phosphorylation of insulin receptor substrate-1 (IRS-1). A self-attenuated mechanism to negatively regulate the function of IRS proteins. J. Biol. Chem. 276:14459-14465.

46. Tobe, K., et al. 2001. Increased expression of the sterol regulatory element-binding protein-1 gene in insulin receptor substrate-2(-/-) mouse liver. J. Biol. Chem. 276:38337-38340. 